\title{
Local toxicity of benzalkonium chloride in ophthalmic solutions following repeated applications
}

\author{
Akihiko Okahara and Kouichi Kawazu \\ Nara Research \& Development Center, Santen Pharmaceutical Co., Ltd., \\ 8916-16 Takayama-cho, Ikoma, Nara 630-0101, Japan
}

(Received April 16, 2013; Accepted May 8, 2013)

\begin{abstract}
We performed repeated toxicity studies of benzalkonium chloride (BAK)-containing vehicles of ophthalmic solutions in monkeys and rabbits to assess the local toxicity of BAK after repeated applications on the ocular surface. Local toxicity of BAK was evaluated by toxicity studies in which a $0.01 \%$ BAK-containing vehicle was applied twice/day for 52 weeks, 4 times/day for 39 weeks, or 6 times/day for 13 weeks, or in which a $0.005 \%$ BAK-containing vehicle was applied 6 times/day for 52 weeks or twice/day for 4 weeks in monkeys. Local toxicity of BAK was also evaluated where a $0.01 \%$ BAK-containing vehicle was applied 6 times/day for 6 weeks, or a $0.005 \%$ BAK-containing vehicle was applied twice/day for 39 weeks or 8 times/day for 4 weeks in rabbits. These doses were chosen because BAK is generally used at concentrations up to $0.01 \%$ in ophthalmic solutions. The BAK-containing vehicle did not cause ophthalmological changes suggestive of irritation, allergy, or corneal damage. We also did not observe any histopathological changes in the eyeball, eyelid, lacrimal gland, and nasal cavity, with repeated applications of BAK for up to 52 weeks, up to 8 times/day, or at concentrations up to $0.01 \%$, in monkeys and rabbits. Our results suggest that BAK in concentrations up to $0.01 \%$ in ophthalmic solution is non-toxic to the eyeball, its accessory organs, and the nasal cavity after long repeated applications.
\end{abstract}

Key words: Benzalkonium chloride, Repeated toxicity, Ophthalmic solution, Eye, Nasal cavity

\section{INTRODUCTION}

Many ophthalmic solutions contain preservatives to prevent contamination by microorganisms. Benzalkonium chloride (BAK) is the most widely used preservative in ophthalmic solutions. Ocular toxicity of BAK has been investigated by many in vitro experiments using cell culture systems, such as human corneal cell lines (Tripathi and Tripathi, 1989; Tripathi et al., 1992; Li and Yan, 2008; Epstein et al., 2009; Ammar et al., 2011), human conjunctival cell lines (De Saint Jean et al., 1999; Debbasch et al., 2000a, 2000b; Brasnu et al., 2008a, 2008b; Epstein et al., 2009; Ammar et al., 2011; Ayaki and Iwasawa, 2011), a three- dimensional model of human corneal epithelium (Pauly et al., 2009; Liang et al., 2011), rabbit corneal cell lines (Cha et al., 2004; Ayaki and Iwasawa, 2011), and bovine corneal cell lines (Ayaki and Iwasawa, 2011). In addition, the ocular toxicity of BAK has been investigated by several in vivo experiments following application on the ocular surface of rats (Becquet et al., 1998), rabbits (Pfister and Burstein, 1976;
Burstein, 1980), and cats (Burstein, 1980). In these studies, BAK was deleterious to corneas and cultured cells at concentrations used clinically. However, it was reported that BAK did not produce significant corneal toxicity in the vast majority of patients by a meta-analysis of randomized, controlled clinical trials of latanoprost ophthalmic solution and timolol ophthalmic solution (Trocme et al., 2010). The levels of BAK contained in ophthalmic solutions are also not likely to cause significant direct toxicity to the corneal epithelium, because there were no significant differences in cytotoxicity between the BAK-containing latanoprost and olopatadine ophthalmic solutions and BAK-free travoprost ophthalmic solution in threedimensional corneal epithelial culture assays (Khoh-Reiter and Jessen, 2009). In addition, some additives in ophthalmic solutions are known to improve BAK-induced corneal epithelial toxicity. For example, there is a remarkable improvement of BAK-induced corneal epithelial toxicity after addition of polyoxyethylene hydrogenated castor oil 40 (HCO-40) to BAK (Uematsu et al., 2011). These studies suggest that BAK concentrations listed on

Correspondence: Akihiko Okahara (E-mail: akihiko.okahara@santen.co.jp) 
a label do not always reflect whether there will be corneal toxicity caused by the ophthalmic solution. Therefore, to determine the toxicity of BAK in clinical practice of ophthalmic solutions, it is necessary to assess the toxicity of BAK in ophthalmic solutions or their vehicles. Furthermore, toxicity of BAK to the eyeball, its accessory organs, and the nasal cavity by repeated applications has not been adequately studied, although many ophthalmic solutions are likely to be repeatedly applied to eyes in clinical use, except a study to investigate toxic effects induced in the rat ocular surface after BAK applications for one month (Becquet et al., 1998).

In this study, to assess local toxicity of BAK contained in ophthalmic solutions following repeated applications on the ocular surface, we investigated the repeated toxicity of BAK-containing vehicles of ophthalmic solutions toward the eyeball, its accessory organs, and the nasal cavity in rabbits and monkeys.

\section{MATERIALS AND METHODS}

BAK-containing vehicles of ophthalmic solutions under development by Santen Pharmaceutical Co., Ltd. (Osaka, Japan) were used as the test articles. Table 1 shows the outline of the toxicity studies of BAK-containing vehicles. The concentration of BAK contained in vehicles was
$0.005 \%(\mathrm{w} / \mathrm{v})$ and $0.01 \%(\mathrm{w} / \mathrm{v})$, because BAK is generally used at concentrations up to $0.01 \%$ in ophthalmic solutions. In monkeys, the local toxicity of BAK was evaluated by $0.01 \%$ BAK-containing vehicle, which was applied twice/day for 52 weeks (experiment 1), 4 times/day for 39 weeks (experiment 2), or 6 times/day for 13 weeks (experiment 3). A $0.005 \%$ BAK-containing vehicle was also applied 6 times/day for 52 weeks (experiment 4) or twice/day for 4 weeks (experiment 5). In rabbits, the local toxicity of BAK was evaluated by $0.01 \%$ BAK-containing vehicle, which was applied 6 times/day for 6 weeks (experiment 6). A $0.005 \%$ BAK-containing vehicle was also applied twice/day for 39 weeks (experiment 7) or 8 times/day for 4 weeks (experiment 8). BAK was obtained from FeF Chemicals A/S (Køge, Denmark). BAK-containing vehicles were supplied from Santen Pharmaceutical Co., Ltd.

All experiments were approved by the Institutional Animal Care and Use Committee, and were performed in accordance with the animal welfare bylaws of Covance Laboratories GmbH (experiment 1), Drug Safety Research Laboratories of Shin Nippon Biomedical Laboratories, Ltd. (experiments 2, 3, 4, 5, 6, and 7), or the Nara Research \& Development Center of Santen Pharmaceutical Co., Ltd. (experiment 8).

All experiments were performed in compliance with

Table 1. Outline of toxicity studies of BAK-containing vehicles of ophthalmic solutions

\begin{tabular}{|c|c|c|c|c|c|c|c|c|}
\hline \multirow{2}{*}{ No. ${ }^{\mathrm{a}}$} & \multirow{2}{*}{$\begin{array}{c}\text { BAK } \\
(\%)\end{array}$} & \multirow{2}{*}{$\mathrm{Nb}^{\mathrm{b}}$} & \multirow{2}{*}{ Durationc } & \multirow{2}{*}{ Freq. ${ }^{\mathrm{d}}$} & \multirow{2}{*}{ Ophthalmol.e } & \multicolumn{2}{|c|}{ Histopathology } & \multirow{2}{*}{$\begin{array}{c}\text { SEM } \\
\text { Cornea }\end{array}$} \\
\hline & & & & & & Eye $^{f}$ & Noseg & \\
\hline \multicolumn{9}{|c|}{ Monkey } \\
\hline 1 & 0.01 & 8 & $52 \mathrm{~W}$ & 2 & $13 \mathrm{~W}, 26 \mathrm{~W}, 39 \mathrm{~W}, 52 \mathrm{~W}$ & $\mathrm{Y}^{\mathrm{h}}$ & & \\
\hline 2 & 0.01 & 8 & $39 \mathrm{~W}$ & 4 & $12 \mathrm{~W}, 25 \mathrm{~W}, 38 \mathrm{~W}$ & Y & & \\
\hline 3 & 0.01 & 6 & $13 \mathrm{~W}$ & 6 & $13 \mathrm{~W}$ & $\mathrm{Y}$ & Y & \\
\hline 4 & 0.005 & 10 & $52 \mathrm{~W}$ & 6 & $12 \mathrm{~W}, 26 \mathrm{~W}, 39 \mathrm{~W}, 52 \mathrm{~W}$ & $\mathrm{Y}$ & $\mathrm{Y}$ & \\
\hline 5 & 0.005 & 10 & $4 \mathrm{~W}$ & 2 & $4 \mathrm{~W}$ & $\mathrm{Y}$ & $\mathrm{Y}$ & $\mathrm{Y}$ \\
\hline \multicolumn{9}{|c|}{$\underline{\text { Rabbit }}$} \\
\hline 6 & 0.01 & 12 & $6 \mathrm{~W}$ & 6 & $3 \mathrm{~W}, 6 \mathrm{~W}$ & $\mathrm{Y}$ & $\mathrm{Y}$ & \\
\hline 7 & 0.005 & 18 & $39 \mathrm{~W}$ & 2 & $13 \mathrm{~W}, 26 \mathrm{~W}, 39 \mathrm{~W}$ & $\mathrm{Y}$ & Y & \\
\hline 8 & 0.005 & 6 & $4 \mathrm{~W}$ & 8 & $1 \mathrm{~W}, 2 \mathrm{~W}, 3 \mathrm{~W}, 4 \mathrm{~W}$ & $\mathrm{Y}$ & $\mathrm{Y}$ & \\
\hline
\end{tabular}

\footnotetext{
a Experiment No.

${ }^{\mathrm{b}}$ Number of animals

${ }^{c}$ Duration of applications $(\mathrm{W}=$ week $)$

${ }^{\mathrm{d}}$ Frequency of applications (time/day)

e The schedule of ophthalmology examination $(\mathrm{W}=$ week $)$

${ }^{\mathrm{f}}$ The eyeball, eyelid and lacrimal gland

g The nasal cavity

${ }^{\text {h }}$ Examined
} 
Repeat toxicity of benzalkonium chloride

Good Laboratory Practice Regulations for Nonclinical Safety Studies on Drugs (Ministry of Health and Welfare, Japan, Ordinance No. 21, March 26, 1997) and OECD Principles of Good Laboratory Practice (revised in 1997) ENV/MC/CHEM (98) 17.

\section{Animals}

\section{Experiment 1}

Three to 4-year-old male and female cynomolgus monkeys (Macaca fascicularis) were supplied by Bioculture Mauritius Ltd. (Rivière des Anguilles, Mauritius). Monkeys were quarantined and acclimatized for approximately 6 weeks prior to commencement of application. The monkeys were individually housed in a room with a temperature of $19-25^{\circ} \mathrm{C}$, relative humidity of $30 \%$ to $70 \%$, 12-hr light/dark cycle, and air ventilation 10 times per hr. They were provided with water ad libitum, and twice daily were given 50 to $70 \mathrm{~g}$ of a commercial pellet diet for primates (Ssniff P10; Ssniff Spezialdiäten GmbH, Soest, Germany). In addition, the monkeys received fresh fruit approximately twice weekly and one slice of bread once weekly.

\section{Experiments 2, 3, 4 and 5}

Three to 7-year-old male and female cynomolgus monkeys (Macaca fascicularis) were supplied by Shin Nippon Biomedical Laboratories, Ltd. (Kagoshima, Japan), Gaoyao Kangda Laboratory Animals Science \& Technology Co., Ltd. (Guangdong, China), Guangzhou Kesen Imports \& Exports Co., Ltd. (Guangzhou, China), Guangxi Grandforest Scientific Primate Company, Ltd. (Guangxi, China), Guangdong Scientific Instruments \& Materials Import/Export Corporation (Guangzhou, China), and China National Scientific Instruments \& Materials Import/Export Corporation (Beijing, China). Monkeys were quarantined and acclimatized for approximately 3 weeks prior to commencement of application, and individually housed in a room with a temperature of $23-29^{\circ} \mathrm{C}$, relative humidity of $35 \%$ to $75 \%$, 12-hr light/ dark cycle, and air ventilation 15 times per hr. They were provided with water ad libitum, and were given approximately $108 \mathrm{~g}$ of solid food (Teklad Global Certified 25\% Protein Primate Diet; Harlan Sprague Dawley Inc., Indianapolis, IN, USA, or HF Primate 5K91 12G 5K9J; Purina Mills, Gray Summit, MO, USA) once daily.

\section{Experiments 6 and 7}

Twelve- to 13-week-old (experiment 6), or 9- to 10-week-old (experiment 7) male and female Dutch rabbits (Kbt: Dutch) were supplied by Biotech Co., Ltd. (Saga, Japan). Rabbits were quarantined and acclima- tized for approximately 3 weeks prior to commencement of application, and individually housed in a room with a temperature of $19-25^{\circ} \mathrm{C}$, relative humidity of $30 \%$ to 70\%, 12-hr light/dark cycle, and air ventilation 15 times per hr. They were provided with water ad libitum, and were given approximately $100 \mathrm{~g}$ of solid food (CR-1; CLEA Japan, Inc., Tokyo, Japan) once daily.

\section{Experiment 8}

Eight-week-old female Japanese white rabbits (Kbl: JW) were supplied by Kitayama Labes Co., Ltd. (Nagano, Japan). Rabbits were quarantined and acclimatized for approximately 2 weeks prior to commencement of application, and individually housed in a room with a temperature of $19-25^{\circ} \mathrm{C}$, relative humidity of $30 \%$ to $70 \%$, 12-hr light/dark cycle, and air ventilation 10 times per hr. They were provided with water ad libitum, and were given approximately $130 \mathrm{~g}$ of solid food (LRC-4; Oriental Yeast Co., Ltd., Tokyo, Japan) once daily.

\section{Administration}

Thirty microliters of $0.01 \%$ BAK-containing vehicle was applied to the ocular surface twice/day (every $12 \mathrm{hr}$ ) for 52 weeks (experiment 1), 4 times/day (every $2 \mathrm{hr}$ ) for 39 weeks (experiment 2), or 6 times/day (every $1.5 \mathrm{hr}$ ) for 13 weeks (experiment 3 ) in monkeys. Thirty microliters of $0.005 \%$ BAK-containing vehicle was applied to the ocular surface 6 times/day (every $1.5 \mathrm{hr}$ ) for 52 weeks (experiment 4) or twice/day (every $12 \mathrm{hr}$ ) for 4 weeks (experiment 5) in monkeys.

Fifty microliters of $0.01 \%$ BAK-containing vehicle was applied on the ocular surface 6 times/day (every $1.5 \mathrm{hr}$ ) for 6 weeks (experiment 6) in rabbits. Fifty microliters of $0.005 \%$ BAK-containing vehicle was applied to the ocular surface twice/day (every $12 \mathrm{hr}$ ) for 39 weeks (experiment 7) or 8 times/day (every $1 \mathrm{hr}$ ) for 4 weeks (experiment 8) in rabbits.

\section{Observations and examinations}

Following applications, ophthalmology (every experiment) and histopathology of the eyeball, eyelid, lacrimal gland (every experiment), and nasal cavity (experiments $3,4,5,6,7,8)$ were performed. The corneal epithelium and endothelium were observed by scanning electron microscopy (SEM) (experiment 5).

\section{Ophthalmology}

In experiment 1 , approximately 2 to $6 \mathrm{hr}$ after the first application on each day, the cornea and conjunctiva were observed using a slit lamp microscope (SL 120; Zeiss Co., Ltd., Jena, Germany), and were evaluated in accord- 
ance with the McDonald-Shadduck method (McDonald and Shadduck, 1977). Corneal epithelia were stained with fluorescein sodium $\left(1.7 \mathrm{mg} / \mathrm{ml}\right.$ Fluoreszein SE Thilo ${ }^{\circledR}$; Alcon Lab., Freiburg, Germany) and observed using a slit lamp microscope for the presence of staining.

In experiment 2, approximately 1 to $3 \mathrm{hr}$ after the last application on each day, the cornea and conjunctiva were observed using a slit lamp microscope (SL-14; Kowa Co., Ltd., Nagoya, Japan), and were evaluated in accordance with the McDonald-Shadduck method. Corneal epithelia were stained with fluorescein paper (Showa Yakuhin Kako Co., Ltd., Tokyo, Japan) and observed using a slit lamp microscope for the presence of staining.

Approximately 2 to $5 \mathrm{hr}$ after the last application on each day in experiments 3, 4, 5 and 6, and at approximately $1 \mathrm{hr}$ after the first application on each day in experiment 7 , the cornea and conjunctiva were observed using a slit lamp microscope, and were evaluated in accordance with the McDonald-Shadduck method. Corneal epithelia were stained with $0.25 \%$ fluorescein sodium (Altaire Pharmaceuticals Inc., Aquebogue, NY, USA) and observed using a slit lamp microscope for the presence of staining.

In experiment 8 , approximately $1 \mathrm{hr}$ after the last application on each day, the cornea and conjunctiva were macroscopically observed, and the ocular irritation scores were recorded according to the modified Draize scoring system (Draize et al., 1944). After macroscopic examination, one drop of $0.5 \%$ fluorescein solution was applied to the cornea of eyes. Corneal epithelia were observed for fluorescein staining using a slit lamp microscope and were evaluated according to the scoring system for fluorescein staining. The $0.5 \%$ fluorescein solution was prepared by dissolving sodium fluorescein (Wako Pure Chemical Industries, Ltd., Osaka, Japan) in saline (Otsuka Pharmaceutical Factory, Inc., Tokyo, Japan).

\section{Histopathology of the eyeball, its accessory organs, and the nasal cavity}

On the day following the end of application, rabbits were anesthetized by an intravenous injection of sodium pentobarbital solution $(64.8 \mathrm{mg} / \mathrm{ml}, 0.7 \mathrm{ml} / \mathrm{kg}$; Tokyo Chemical Industry Co., Ltd., Tokyo, Japan, or $50 \mathrm{mg} / \mathrm{ml}$, $0.5 \mathrm{ml} / \mathrm{kg}$; Dainippon Pharmaceutical Co., Ltd., Osaka, Japan) into the auricular vein, and euthanized by exsanguination. Monkeys were anesthetized by an intravenous injection of sodium pentobarbital solution $(64.8 \mathrm{mg} / \mathrm{ml}$, $0.4 \mathrm{ml} / \mathrm{kg}$; Tokyo Chemical Industry Co., Ltd., Tokyo, Japan) into the cephalic vein of the forearm, and euthanized by exsanguination. Eyeballs (including the optic nerve and bulbar conjunctiva) were fixed in Davidson's fixation solution (experiment 1), in a solution of $3 \%$ glu- taraldehyde and $2.5 \%$ paraformaldehyde (experiments $2,3,4,5,6$ and 7), or in F-G fixation solution (experiment 8). Eyelids (including palpebral conjunctiva), lacrimal glands, and nasal cavities were fixed in 10\% neutral buffered formalin. Organs and tissues were trimmed, embedded in paraffin, sectioned, stained with hematoxylin-eosin, and were examined microscopically.

\section{Observation of corneal epithelium and endothelium by SEM}

Eyes of each animal were removed and fixed by submersion in a solution of $2.5 \%$ paraformaldehyde and $3 \%$ glutaraldehyde (experiment 5). From fixed eye balls, corneas were excised, rinsed and postfixed in $1 \%$ buffered osmium tetroxide solution. After dehydration in ethanol at increasing concentrations, critical point drying and gold evaporation were conducted on the tissues. The corneal epithelium and endothelium were viewed using SEM (JSM-5200; JEOL Co., Ltd., Tokyo, Japan).

\section{RESULTS}

After application of $0.01 \%$ BAK-containing vehicle twice/day for 52 weeks (experiment 1), and 4 times/day for 39 weeks in monkeys (experiment 2), there were no changes in the ophthalmology and histopathology of the eyeball, eyelid, and lacrimal gland (Table 2). Application of $0.01 \%$ BAK-containing vehicle 6 times/day for 13 weeks in monkeys (experiment 3 ) induced no changes in the ophthalmology and histopathology of the eyeball, eyelid, lacrimal gland, and nasal cavity (Table 2). Following application of $0.005 \%$ BAK-containing vehicle 6 times/day for 52 weeks (experiment 4), and twice/day for 4 weeks (experiment 5) in monkeys, we observed no changes in the ophthalmology and histopathology of the eyeball, eyelid, lacrimal gland, and nasal cavity (Table 3 ). In addition to these results, in experiment 5 , there were no changes in the epithelium and endothelium of corneas as shown by SEM.

Application of $0.01 \%$ BAK-containing vehicle 6 times/day for 6 weeks (experiment 6), and application of $0.005 \%$ BAK-containing vehicle twice/day for 39 weeks (experiment 7) and 8 times/day for 4 weeks (experiment 8 ) in rabbits did not change the ophthalmology and histopathology of the eyeball, eyelid, lacrimal gland, and nasal cavity (Table 4).

\section{DISCUSSION}

In the present study, we assessed the toxicity of $0.005 \%$ and $0.01 \%$ BAK-containing vehicles of ophthalmic solutions in rabbits and monkeys, for an application duration 
Repeat toxicity of benzalkonium chloride

Table 2. Toxicity of $0.01 \%$ BAK-containing vehicle of ophthalmic solution in monkeys

\begin{tabular}{|c|c|c|c|}
\hline Experiment No. & 1 & 2 & 3 \\
\hline Duration $^{\mathrm{a}}$ & $52 \mathrm{~W}$ & $39 \mathrm{~W}$ & $13 \mathrm{~W}$ \\
\hline Frequency ${ }^{b}$ & 2 & 4 & 6 \\
\hline Number of animals & 8 & 8 & 6 \\
\hline Ophthalmology & Normal & Normal & Normal \\
\hline \multicolumn{4}{|l|}{ Histopathology } \\
\hline Eyeball & Normal & Normal & Normal \\
\hline Eyelid & Normal & Normal & Normal \\
\hline Lacrimal gland & Normal & Normal & Normal \\
\hline Nasal cavity & $\mathrm{NE}^{\mathrm{c}}$ & NE & Normal \\
\hline
\end{tabular}

${ }^{\text {a }}$ Duration of applications $(\mathrm{W}=$ week $)$

${ }^{\mathrm{b}}$ Frequency of applications (time/day)

c Not examined

Table 3. Toxicity of $0.005 \%$ BAK-containing vehicle of ophthalmic solution in monkeys

\begin{tabular}{lcc}
\hline Experiment No. & 4 & 5 \\
\hline Duration $^{\mathrm{a}}$ & $52 \mathrm{~W}$ & $4 \mathrm{~W}$ \\
Frequency $^{\mathrm{b}}$ & 6 & 2 \\
Number of animals & 10 & 10 \\
\hline Ophthalmology & Normal & Normal \\
Histopathology & & \\
$\quad$ Eyeball & Normal & Normal \\
$\quad$ Eyelid & Normal & Normal \\
$\quad$ Lacrimal gland & Normal & Normal \\
$\quad$ Nasal cavity & Normal & Normal \\
SEM & & \\
$\quad$ Epithelium & $\mathrm{NE}$ & Normal \\
Endothelium & $\mathrm{NE}$ & Normal \\
\hline
\end{tabular}

${ }^{a}$ Duration of applications ( $\mathrm{W}=$ week $)$

${ }^{\mathrm{b}}$ Frequency of applications (time/day)

c Not examined

of 4-52 weeks and a frequency of 2-8 times/day. These doses were chosen because BAK is generally used at concentrations up to $0.01 \%$ in ophthalmic solutions. The toxicity was assessed using ophthalmology and histopathology of the eyeball and its accessory organs, such as the eyelids and lacrimal gland, as well as SEM examination of the corneal epithelium and endothelium. The toxicity of BAK to the nasal cavity was also histopathologically assessed, because there is continuous flow due to drainage through the nasolacrimal canal (Chrai et al., 1973). As a result of this investigation, BAK-containing vehicle did not cause ophthalmological changes suggestive of irritation, or corneal damage. BAK-containing vehicle also did not cause histopathological changes in the eyeball, its accessory organs, and the nasal cavity with repeated applications up to 52 weeks and up to 8 times/day, and using concentrations up to $0.01 \%$. There are several reasons why the concentration of $0.01 \%$, at which BAK was deleterious in previous studies about the BAK-induced toxicity (Pfister and Burstein, 1976; Burstein, 1980; Tripathi and Tripathi, 1989; Tripathi et al., 1992; Becquet et al., 1998; De Saint Jean et al., 1999; Debbasch et al., 2000a, 2000b; Cha et al., 2004; Brasnu et al., 2008a, 2008b; Li and Yan, 2008; Epstein et al., 2009; Pauly et al., 2009; Ammar et al., 2011; Ayaki and Iwasawa, 2011; Liang et al., 2011), was non-toxic in this study. One of the reasons could be because the concentration of the drug in the tear film following ocular application can be greatly affected by several factors. The most significant factor reducing the drug concentration in the tear film has been shown to be instilled drug solution drainage away from the precorneal area through the nasolacrimal canal (Chrai et al., 1973). The physiological turnover rate of tear fluid is approximately $16 \%$ per minute in humans (Mishima et al., 1966), and is increased because of stimulation resulting from drop instillation (Shell, 1982). The instilled dose leaves the precorneal area within 2 minutes of application in humans (Eaga et al., 2009), and 5 to 10 min in rabbits (Chrai et al., 1973). Most of the applied eye drops are lost to drainage in the first 15 to $30 \mathrm{sec}$ after application in humans (Shell, 1982). Therefore, the contact time of BAK with the cornea in vivo is speculated to be shorter than that of in vitro studies, which could one of the reasons why the toxicity of BAK in the present study 


\section{A. Okahara and K. Kawazu}

Table 4. Toxicity of BAK-containing vehicle of ophthalmic solution in rabbits

\begin{tabular}{lccc}
\hline Experiment No. & 6 & 7 & 8 \\
\hline BAK(\%) & 0.01 & 0.005 & 0.005 \\
Duration $^{\text {a }}$ & $6 \mathrm{~W}$ & $39 \mathrm{~W}$ & $4 \mathrm{~W}$ \\
Frequency & 6 & 2 & 8 \\
Number of animals & 12 & Normal & Normal \\
\hline Ophthalmology & Normal & & Normal \\
Histopathology & & Normal & Normal \\
$\quad$ Eyeball & Normal & Normal & Normal \\
\multicolumn{1}{l}{ Eyelid } & Normal & Normal & Normal \\
\hline
\end{tabular}

a Duration of applications $(\mathrm{W}=$ week $)$

${ }^{\mathrm{b}}$ Frequency of applications (time/day)

was weaker than that of in vitro studies. Another reason why the toxicity of BAK in the present study was weaker than that in previous studies about the BAK-induced toxicity could be because vehicles of ophthalmic solutions were used as the basic solution for dissolving BAK in this study. It was reported that $0.01 \%$ BAK dissolved in $0.9 \%$ saline or phosphate-buffered saline showed corneal and/or conjunctival damage in rabbits (Pfister and Burstein, 1976; Burstein, 1980), cats (Burstein, 1980) or rats (Becquet et al., 1998). Meanwhile, some additives in ophthalmic solution are known to improve BAK-induced toxicity. For example, HCO-40 prevents BAK-induced corneal toxicity and cytotoxicity to corneal epithelial cells (Uematsu et al., 2011). Protective effects against BAKinduced cytotoxicity were also reported for D-mannitol using corneal epithelial cells (Nagai et al., 2011). However, it was unlikely that additives in vehicles prevented BAK-induced toxicity in the present study, because vehicles used in the present study did not contain HCO-40 and D-mannitol.

Many ophthalmic drugs induce allergic reactions, such as allergic contact dermatitis of the periorbital region or immediate hypersensitivity reactions (Baudouin, 2005; Jappe et al., 2006; Spaeth, 2006; Ventura et al., 2006; Chaudhari and Maibach, 2007; Uter et al., 2009). In a previous study, among 332 patients patch-tested with their own topical anti-glaucoma eye drops because of suspected allergic contact dermatitis, a total of $43 / 332$ (12.95\%) showed a positive patch test reaction (Jappe et al., 2006). Allergic reactions can be provoked by additives, as well as active ingredients. Analysis of clinical patch test data of patients with $0.1 \%$ BAK in petrolatum in three dif- ferent series (topical drugs, ophthalmics, and disinfectants) suggests that BAK is a contact allergen (Uter et al., 2008). In addition, a $0.5 \%$ or higher BAK concentration elicits dose-responsive and statistically significant lymph node proliferation in the murine local lymph node assay, which is an assay for identifying chemicals as potential human sensitizers (Woolhiser et al., 1998). However, in the present study, BAK-containing vehicle caused no ocular, periocular, or systemic allergies with repeated applications up to 52 weeks at concentrations up to $0.01 \%$. Our results suggest that $\mathrm{BAK}$ does not induce allergic reactions at low concentrations, such as $0.005 \%$ and $0.01 \%$, which are generally used concentrations in ophthalmic solutions.

In conclusion, our results suggest that BAK in concentrations up to $0.01 \%$ in ophthalmic solutions is non-toxic to the eyeball, its accessory organs, and the nasal cavity after long repeated applications.

\section{REFERENCES}

Ammar, D.A., Noecker, R.J. and Kahook, M.Y. (2011): Effects of benzalkonium chloride- and polyquad-preserved combination glaucoma medications on cultured human ocular surface cells. Adv. Ther., 28, 501-510.

Ayaki, M. and Iwasawa, A. (2011): Cell viability of four corneoconjunctival cell lines exposed to five preservatives and a surfactant used for infection control in eyedrops. Biocontrol. Sci., 16, 117-121.

Baudouin, C. (2005): Allergic reaction to topical eyedrops. Curr. Opin. Allergy Clin. Immunol., 5, 459-463.

Becquet, F., Goldschild, M., Moldovan, M.S., Ettaiche, M., Gastaud, P. and Baudouin, C. (1998): Histopathological effects of topical ophthalmic preservatives on rat corneoconjunctival surface. Curr. Eye Res., 17, 419-425. 
Repeat toxicity of benzalkonium chloride

Brasnu, E., Brignole-Baudouin, F., Riancho, L., Guenoun, J.M., Warnet, J.M. and Baudouin, C. (2008a): In vitro effects of preservative-free tafluprost and preserved latanoprost, travoprost, and bimatoprost in a conjunctival epithelial cell line. Curr. Eye Res., 33, 303-312.

Brasnu, E., Brignole-Baudouin, F., Riancho, L., Warnet, J.M. and Baudouin, C. (2008b): Comparative study on the cytotoxic effects of benzalkonium chloride on the Wong-Kilbourne derivative of Chang conjunctival and IOBA-NHC cell lines. Mol. Vis., 14, 394-402.

Burstein, N.L. (1980): Preservative cytotoxic threshold for benzalkonium chloride and chlorhexidine digluconate in cat and rabbit corneas. Invest. Ophthalmol. Vis. Sci., 19, 308-313.

Cha, S.H., Lee, J.S., Oum, B.S. and Kim, C.D. (2004): Corneal epithelial cellular dysfunction from benzalkonium chloride (BAC) in vitro. Clin. Exp. Ophthalmol., 32, 180-184.

Chaudhari, P.R. and Maibach, H.I. (2007): Allergic contact dermatitis from ophthalmics. Contact Dermatitis, 57, 11-13.

Chrai, S.S., Patton, T.F., Mehta, A. and Robinson, J.R. (1973): Lacrimal and instilled fluid dynamics in rabbit eyes. J. Pharm. Sci., 62, 1112-1121.

De Saint Jean, M., Brignole, F., Bringuier, A.F., Bauchet, A., Feldmann, G. and Baudouin, C. (1999): Effects of benzalkonium chloride on growth and survival of Chang conjunctival cells. Invest. Ophthalmol. Vis. Sci., 40, 619-630.

Debbasch, C., Rat, P., Warnet, J.-M., De Saint Jean, M., Baudouin, C. and Pierre-Jean, P. (2000a) Evaluation of the toxicity of benzalkonium chloride on the ocular surface. J. Toxicol. Cut. Ocular Toxicol., 19, 105-115.

Debbasch, C., De Saint Jean, M., Pisella, P.-J., Rat, P., Warnet, J.M. and Baudouin, C. (2000b): Influence of preservatives on conjunctival cells in vitro. J. Toxicol. Cut. Ocular Toxicol., 19, 79-88.

Draize, J.H., Woodard, G. and Calvery, H.O. (1944): Methods for the study of irritation and toxicity of substances applied topically to the skin and mucous membranes. J. Pharmacol. Exp. Ther., 82, 377-390.

Eaga, C.M., Kandukuri, J.M., Allenki, V. and Yamsani, M.R. (2009): In-situ gels -a novel approach for ocular drug delivery. Der. Pharmacia Lettre, 1, 21-33.

Epstein, S.P., Ahdoot, M., Marcus, E. and Asbell, P.A. (2009): Comparative toxicity of preservatives on immortalized corneal and conjunctival epithelial cells. J. Ocul. Pharmacol. Ther., 25, 113119.

Jappe, U., Uter, W., Menezes de Pádua, C.A., Herbst, R.A. and Schnuch, A. (2006): Allergic contact dermatitis due to betablockers in eye drops: a retrospective analysis of multicentre surveillance data 1993-2004. Acta Derm. Venereol., 86, 509-514.

Khoh-Reiter, S. and Jessen, B.A. (2009): Evaluation of the cytotoxic effects of ophthalmic solutions containing benzalkonium chloride on corneal epithelium using an organotypic 3-D model. BMC Ophthalmol., 9, 5.

Li, J. and Yan, X. (2008): Toxicity of benzalkonium chloride on human corneal epithelial cells in vitro. Chin. Ophthal. Res., 26, 814-817.

Liang, H., Pauly, A., Riancho, L., Baudouin, C. and BrignoleBaudouin, F. (2011): Toxicological evaluation of preservative- containing and preservative-free topical prostaglandin analogues on a three-dimensional-reconstituted corneal epithelium system. Br. J. Ophthalmol., 95, 869-875.

McDonald, T.O. and Shadduck, J.A. (1977): In Dermatoxicology and Pharmacology, p.139, John Wiley \& Sons, New York.

Mishima, S., Gasset, A., Klyce, S.D.Jr. and Baum, J.L. (1966): Determination of tear volume and tear flow. Invest. Ophthalmol., 5, 264-276.

Nagai, N., Murao, T., Oe, K., Ito, Y., Okamoto, N. and Shimomura, Y. (2011): In vitro evaluation for corneal damages by anti-glaucoma combination eye drops using human corneal epithelial cell (HCE-T). YAKUGAKU ZASSHI, 131, 985-991.

Pauly, A., Meloni, M., Brignole-Baudouin, F., Warnet, J.M. and Baudouin, C. (2009): Multiple endpoint analysis of the 3D-reconstituted corneal epithelium after treatment with benzalkonium chloride: Early detection of toxic damage. Invest. Ophthalmol. Vis. Sci., 50, 1644-1652.

Pfister, R.R. and Burstein, N. (1976): The effects of ophthalmic drugs, vehicles, and preservatives on corneal epithelium: a scanning electron microscope study. Invest. Ophthalmol., 15, 246259.

Shell, J.W. (1982): Pharmacokinetics of topically applied ophthalmic drugs. Surv. Ophthalmol., 26, 207-218.

Spaeth, G.L. (2006): Allergic contact dermatitis caused by topical eye drops. Am. J. Ophthalmol., 142, 706.

Tripathi, B.J. and Tripathi, R.C. (1989): Cytotoxic effects of benzalkonium chloride and chlorobutanol on human corneal epithelial cells in vitro. Lens Eye Toxic. Res., 6, 395-403.

Tripathi, B.J., Tripathi, R.C. and Kolli, S.P. (1992): Cytotoxicity of ophthalmic preservatives on human corneal epithelium. Lens Eye Toxic Res., 9, 361-375.

Trocme, S., Hwang, L.J., Bean, G.W. and Sultan, M.B. (2010): The role of benzalkonium chloride in the occurrence of punctate keratitis: A meta-analysis of randomized, controlled clinical trials. Ann. Pharmacother., 44, 1914-1921.

Uematsu, M., Kumagami, T., Shimoda, K., Kusano, M., Teshima, M., To, H., Kitahara, T., Kitaoka, T. and Sasaki, H. (2011): Polyoxyethylene hydrogenated castor oil modulates benzalkonium chloride toxicity: Comparison of acute corneal barrier dysfunction induced by travoprost $\mathrm{z}$ and travoprost. J. Ocul. Pharmacol. Ther., 27, 437- 444.

Uter, W., Lessmann, H., Geier, J. and Schnuch, A. (2008): Is the irritant benzalkonium chloride a contact allergen? A contribution to the ongoing debate from a clinical perspective. Contact Dermatitis, 58, 359-363.

Uter, W., Menezes de Pádua, C., Pfahlberg, A., Nink, K., Schnuch, A. and Behrens-Baumann, W.. (2009): Contact allergy to topical ophthalmological drugs - epidemiological risk assessment. Klin. Monbl. Augenheilk, 226, 48-53.

Ventura, M.T., Viola, M., Gaeta, F., Di Leo, E., Buquicchio, R. and Romano, A. (2006): Hypersensitivity reactions to ophthalmic products. Curr. Pharm. Des., 12, 3401-3410.

Woolhiser, M.R., Hayes, B.B. and Meade, B.J. (1998): A combined murine local lymph node and irritancy assay to predict sensitization and irritancy potential of chemicals. Toxicology Methods, 8, 245-256. 